\title{
Airborne EM Footprints
}

David Beamish

British Geological Survey, Keyworth, Nottingham, NG12 5GG, UK

Beamish, D. Airborne EM footprints. Geophysical Prospecting, 2003, 51, 1-12.

DOI: $\underline{10.1046 / j .1365-2478.2003 .00353 . x}$

\section{Corresponding author:}

David Beamish

British Geological Survey, Keyworth, Nottingham, NG12 5GG, UK

Email: D.Beamish@bgs.ac.uk.

Tel: 01159363432

Fax: 01159363261

Keywords: Electromagnetic, airborne, frequency domain, modelling 


\section{ABSTRACT}

As frequency domain airborne electromagnetic studies (AEM) move towards more detailed assessments of the near surface, the behaviour of system footprints, and hence the spatial averages involved in the measurement, becomes important. Published estimates suffer from two main limitations; firstly they are based on perfectly conducting, thin sheet models and, secondly, they are system specific. The present study is a revision of footprint estimates based on (i) a finitely conducting half-space and (ii) an at-surface scale estimate that uses the spatial equivalent of the conventional electromagnetic skin depth.

In order to remove the system dependence, a transmitter footprint is defined in terms of electromagnetic skin distance. Only the limiting cases of vertical and horizontal magnetic dipole sources then require analysis. Electromagnetic skin distances, two for each of the coil orientations, are defined. The revised definition makes it possible to investigate the footprint behaviour of both towed-bird and fixed-wing AEM systems over an altitude range from 20 to $100 \mathrm{~m}$. The footprint/altitude ratio has a primary dependence on altitude and a secondary dependence on both resistivity and frequency. The analysis covers a frequency range from 1 to $100 \mathrm{kHz}$ and results are presented for two specific resistivity values that represent conductive $(10 \Omega \mathrm{m})$ and resistive $(1000 \Omega \mathrm{m})$ environments.

The revised footprint parameters display a quasi-linear behaviour with altitude, particularly for mid-range frequencies. This behaviour enables the coefficients of linear, least-squares relationships to be obtained thus assisting with the prediction of footprint estimates for survey planning and interpretation. A comparison of the new estimates with published values suggests that existing footprint values for a vertical magnetic dipole should be revised downward. 


\section{INTRODUCTION}

Airborne electromagnetic (AEM) measurements, traditionally used for mineral exploration, are increasingly being applied to a variety of near surface uses. Recent applications of frequency-domain AEM include infrastructure (Beard and Lutro, 2000), groundwater and groundwater vulnerability (Fitterman \& Deszcz-Pan, 1998; Kirsch et al. 2001), environmental surveys (Jokinen and Lanne, 1996; Doll et al., 2000; Beamish and Kurimo, 2000) and Quaternary and surficial geological mapping (Puranen et al., 1999). The frequency-domain systems, discussed here, exist as towed-bird helicopter configurations (HEM systems) and as fixed-wing (wing-tip sensor) configurations. Typically HEM operates the towed sensor bird about $30 \mathrm{~m}$ above ground level while fixed-wing systems (with larger dipole moments) may be flown much higher while maintaining adequate signal/noise.

EM induction by elevated magnetic dipoles is governed both by the frequency/bandwidth and the geometrical attributes of the system, including sensor height. The underlying coil-coil orientation issue has been discussed by a number of authors (Fraser, 1979; Sengpiel, 1983; Liu and Becker, 1990). The three coil-coil systems discussed here are shown schematically in Figure 1. The first two systems employ vertical coil arrangements and use a horizontal magnetic dipole (HMD) transmitter. The third system employs a pair of horizontal coplanar coils and uses a vertical magnetic dipole (VMD) transmitter.

Modern towed birds provide a bandwidth from several hundred Hertz to over 100 $\mathrm{kHz}$ using up to 5 pairs of transmitting and receiving coils. Coil separations are usually between 5 and $8 \mathrm{~m}$. Some bird systems use only horizontal coplanar coil pairs (Figure 1c). Vertical coil pairs in towed birds are invariably in a coaxial arrangement (Figure 1a) along the axis of the bird. The only, fully operational, fixed-wing AEM system is that operated by the Geological Survey of Finland (Poikonen et al, 1998). The coils are wing-tip mounted (separation of $21.4 \mathrm{~m}$ ) and are vertical coplanar (Figure 1b). Coupling ratios at two frequencies $(3.1$ and $14.4 \mathrm{kHz}$ ) are recorded 
simultaneously at $4 \mathrm{~Hz}$. Coupling ratios are here defined as the secondary to primary field ratio, multiplied by $10^{6}$, for both in-phase and in-quadrature components.

When considering detailed near surface applications, an assessment of the AEM system footprint (i.e. the at-surface lateral scale influencing the measurement) becomes an important issue. Resistivity contrasts exist at a wide range of scales. Well populated areas such as the UK are characterised by a considerable redistribution and reworking of at-surface geological materials (road construction is just one pervasive example). "Geometrically imposed" AEM induced electric fields have both a maximum amplitude and maximum physical scale at the surface. It is natural to expect an extensive interaction between at and near surface components of both rural and urban environments with AEM measurements. Knowledge of the behaviour of the AEM footprint/altitude ratio is thus important for survey design in relation to both target discrimination and flight line spacing.

Liu and Becker (1990) and Kovacs et al. (1995) have discussed the footprint/altitude ratio for helicopter EM systems and measurements over sea ice. The model used was a sheet of infinite conductivity taken to represent the surface of the seawater. The authors defined the footprint of an AEM system as the side of a square surface, centred directly below the transmitter coil, that contains the induced currents which account for $90 \%$ of the observed secondary magnetic field. Obviously, using this definition, the footprint depends on the specific system used (the coil orientations and separation) and the sensor height. The footprint models are clearly restrictive on two main accounts. Firstly, the model is based on an infinitely conducting thin sheet so that any frequency-dependence is not taken into account. The results also depend on the AEM system considered, in particular, the coil separation which differs substantially between towed-bird and fixed-wing systems.

An alternative study of AEM footprints is conducted here. The model used is an elevated magnetic dipole transmitter above a finitely conducting half-space (3D source with 1D earth). This model provides a volumetric footprint but only the atsurface footprints are analysed in detail. In order to remove the system dependence 
of previous studies, a transmitter footprint is defined in terms of the electromagnetic "skin distance" which has the same attributes laterally as the conventional skin depth of an electromagnetic field. Only the limiting cases of a vertical transmitter coil (a HMD) and a horizontal transmitter coil (a VMD) then require study.

Electromagnetic skin distances, two for each of the coil orientations, are found to provide adequate footprint parameters. These at-surface footprints have been analysed as a function of transmitter altitude. The behaviour has a primary dependence on altitude and a secondary dependence on both resistivity and frequency. The behaviour is studied across the altitude range from 20 to $100 \mathrm{~m}$ thus covering a typical range of towed-bird and fixed wing systems. The analysis covers a frequency range of $1 \mathrm{kHz}$ to $100 \mathrm{kHz}$ and results are presented for two specific resistivity values that represent conductive $(10 \Omega \mathrm{m})$ and resistive $(1000 \Omega \mathrm{m})$ environments.

\section{METHODOLOGY}

The electromagnetic solution for an elevated magnetic dipole above a homogenous earth was developed by Wait $(1954,1955)$. The model was extended to the 2-layer case by Frischknecht (1967). Anderson (1979) and Siemon (2001) discuss subsequent generalisation to a layered half space. The frequency domain electromagnetic vector potentials can be used to obtain the quasi static electric and magnetic fields within the half-space. Here, although the frequency analysis extends to $100 \mathrm{kHz}$, quasi static (low frequency) behaviour is assumed.

Following Ward (1967), the expressions for the total (free space primary and secondary) electric field within a half-space $(z \geq 0)$ in the plane $y=0$ are given by :

$$
E_{y}=-\frac{i \omega \mu M}{2 \pi} \int_{0} \frac{\lambda^{2}}{\lambda+m} e^{-\lambda h} e^{-m z} J_{1}(\lambda x) d \lambda
$$


for a VMD in the $+z$ direction, and

$$
\left.\begin{array}{c}
E_{y}=-\frac{i \omega \mu M}{2 \pi}\left\{\frac{1}{x} \int_{0}^{\infty} \frac{\lambda^{2}}{\lambda+m} e^{-\lambda h} e^{-m z} J_{1}(\lambda x) d \lambda\right. \\
+\int_{0}^{\infty} \frac{\lambda^{2}}{\lambda+m} e^{-\lambda h} e^{-m z} J_{0}(\lambda x) d \lambda
\end{array}\right\}
$$

for a HMD in the $+x$ direction, and where $m=v\left(\lambda^{2}-i \omega \mu \sigma\right), \sigma$ being the conductivity of the half-space. In these expressions the dipole is at a height $h$ above the surface of the half-space, $z$ is the depth below the surface, $M$ is the dipole moment and $J_{0}(\lambda x)$ and $J_{1}(\lambda x)$ are Bessel functions of the first kind of orders 0 and 1 , respectively.Accurate techniques for the numerical integration of the expressions have been established (Frischknecht, 1967, Christensen, 1990). Here the Hankel transforms in equations (1) and (2) are evaluated using the filter coefficients of Anderson (1975). Reid and Macnae (1999) discuss equivalent, electric field calculations in relation to a similar problem to the one considered here. Non-planar, or local source (HMD and VMD magnetic dipoles), skin depths were investigated by Reid and Macnae (1999) for large-scale geophysical systems. Reid and Macnae (1999) compared local source with plane wave skin depths. Reid and Macnae (1999) calculated the total electric field on a regular grid within the half-space, and then determined the depth at which the amplitude of the field within the earth fell below 1/e of its value at the surface. Typical grid intervals used for the calculations were 10 $\mathrm{m}$ in the horizontal direction and $1 \mathrm{~m}$ in the vertical.

As discussed later, the problem considered here is the determination of an atsurface, lateral skin distance (a footprint) for specific source dipole orientations. 
Again the electric field is estimated within a half-space, largely for simplicity. Here the calculation of the total induced electric field has been achieved using a $1 \mathrm{~m}$ grid in the horizontal plane. The anticipated accuracy in the estimates of footprint sizes is expected to be of the same order as the grid (i.e. about $1 \mathrm{~m}$ ).

\section{EXISTING AEM SYSTEM FOOTPRINTS}

Induced AEM current distributions on a planar surface were presented by Liu and Becker (1990) who described methods for the determination of sea-ice thickness. The model used was a sheet of infinite conductivity taken to represent the surface of the seawater. Clearly in this case, the model of induced currents operates at the inductive limit and any defined footprints are confined to a surface rather than a volume. The authors, for ease of numerical computation, defined the footprint of an AEM system as the side of a square surface, centred directly below the transmitter coil, that contains the induced currents which account for $90 \%$ of the observed secondary magnetic field (Liu and Becker, 1990). Obviously, using this definition, the footprint depends on the specific system used (the coil orientations and separation) and the sensor height. The adopted definition, although somewhat arbitrary, is straightforward in that the zone in which the greatest current flows will be the region that contributes most to the response measured by the AEM system.

The AEM systems considered by Liu and Becker (1990) were components of the same towed-bird helicopter instrument. The two coil-coil orientations involved were horizontal coplanar (a VMD system) and vertical coaxial (a HMD system). The separation of the coil-coil pairs was $6.5 \mathrm{~m}$. The footprints of the two systems were calculated as continuous contributions to the secondary field at the receiver, as a function of altitude. Applying the $90 \%$ definition it was found that the footprint of the coaxial system is a square with a side length of 1.35 times the sensor elevation. Thus for a typical bird height of $30 \mathrm{~m}$, it is a square with $40 \mathrm{~m}$ sides. For the coplanar system, the relative contribution grows more slowly with altitude and hence 
produces a larger footprint. The footprint of this system was found to be 3.73 times the sensor elevation.

Kovacs et al. (1995) also made use of the Liu and Becker footprint model, and reiterated it, when discussing the footprint/altitude ratio for helicopter electromagnetic sounding of sea-ice thickness. Their findings were that, for a vertical coaxial coil arrangement, the apparent footprint diameter was about 1.3 times the sensor height above the sea-ice interface and for a horizontal coplanar coil configuration the ratio was about 3.8 times the sensor height.

The footprint models discussed above are clearly restrictive on two main accounts. Firstly, the model is based on an infinitely conducting thin sheet so that any frequency-dependence is not allowed for and, in addition, the footprint is confined to a surface effect. Secondly, the results discussed depend on the AEM system considered, in particular, the coil separation which differs substantially between towed-bird and fixed-wing systems. The basic definition of the footprint uses the secondary, received fields and these coupling ratios depend on both the coil orientations and coil separation used in the calculation.

As an example, the coupling ratios for the three most common AEM configurations (vertical coaxial, vertical coplanar and horizontal coplanar) have been calculated for a fixed-wing system (coil separation of $20 \mathrm{~m}$ ) and a towed-bird system (coil separation of $6 \mathrm{~m}$ ) for a series of half-space models. The results for an elevation of $40 \mathrm{~m}$ and a frequency of $3 \mathrm{kHz}$ are compared in Figure 2, across a range of half-space resistivities from 0.001 to $10000 \Omega \mathrm{m}$. The results essentially define the system sensitivities for the 'lower frequency end' of typical AEM bandwidths. The height may be slightly 'high' for a towed-bird system (a $30 \mathrm{~m}$ bird height is common) and slightly low for fixed-wing systems (although this survey height has been used both in Finland and the U.K.).

In terms of the three systems considered, the degree of coupling is found to vary systematically as VMD (coplanar) > HMD (coplanar) > HMD (coaxial). As an example, 
the modulus of the $20 \mathrm{~m}$ separation coupling ratios obtained for a resistivity of 100 $\Omega \mathrm{m}$ are 2794, 1435 and $679 \mathrm{ppm}$ for each of the three systems. The equivalent values obtained for the $6 \mathrm{~m}$ separation analysis also decrease systematically, by over an order of magnitude, to values of 79,40 and 20 ppm, respectively.

In Figure 2, the in-phase components (lines with no symbols) of all three systems saturate towards low resistivities and diagnostic information is then contained in the quadrature response (lines with symbols). The peak sensitivity, largely determined by the quadrature response, for the $3 \mathrm{kHz}$ frequency occurs at just less than $10 \Omega \mathrm{m}$ in all three systems. In resistive environments both in-phase and quadrature components are reduced so that the system noise floor (say between 1 and several ppm) inevitably becomes an issue. The modelling of AEM footprints, discussed above, was performed at the inductive limit (limit of high frequency and/or low resistivity in Figure 2) so corresponds to a saturated in-phase response and zero quadrature response.

The restrictions of the existing AEM analyses of footprint/altitude behaviour can be overcome by ignoring the coupling effects at the receiver which depend on, among other things, the coil separation and concentrating instead on the behaviour of the induced current distribution generated by the transmitter. The fields of an AEM transmitter can be calculated throughout a simple model made up of two halfspaces comprising air above a uniform earth. The footprint, in terms of this form of modelling (3D source and 1D earth), actually becomes a volume in the lower halfspace. The spatial distribution of the induced electric field is governed by the orientation and altitude of the transmitter. The maximum electric fields are produced at the surface. Within the earth these fields decay and form a volumetric footprint (with maximum lateral extent at the surface) that fully defines the principal sensitivity zone of the measurement. It is this principal volume that will determine the extent to which any 2D and 3D variations in resistivity structure, at a given physical scale, will influence the measurement. 
The transmitter footprint, a physical scale length, is conveniently defined in terms of the traditional electromagnetic scale-length parameter of skin depth. The skin depth is employed in EM systems to estimate the depth of penetration, usually of plane wave fields (Spies, 1989). The skin depth $(\delta)$ is defined as the depth at which the amplitude of an induced electric field falls to 1 /e (about $37 \%$ ) of its surface value.For our purposes, when dealing with non-planar fields, we can usefully replace the word 'depth' by 'distance' and precede the word 'surface' by 'maximum'. Thus for a given transmitter orientation, there exists a position or locus of positions of maximum induced field, at the surface, which is geometrically controlled i.e. it is stationary for a HMD source and is a function of height for a VMD source. By estimating a 'skin distance' away from the maximum, a principal zone of influence (a footprint) can be estimated using a well established parameter of electromagnetic induction.

Geometrical, or local source, skin depths were investigated by Reid and Macnae (1999) for large-scale (meaning large source-receiver separation) geophysical systems. The authors compared local source with plane wave skin depths and found that close to the transmitter (small offsets), the local source skin depth is less than the plane wave skin depth. With increasing offset from the source, the local source skin depth increases to a maximum value of almost twice the plane wave skin depth and then decreases until it becomes equal to the plane wave skin depth at large offsets.

\section{AEM TRANSMITTER FOOTPRINTS}

In considering only footprints from the transmitter, the issues of different sourcereceiver configurations are simplified. Essentially, we have only the limiting cases of a horizontal magnetic dipole (HMD) and a vertical magnetic dipole (VMD) source to consider. Schematic cross sections of the behaviour of the primary (transmitted) field of these two principal transmitter types were published by Fraser (1979, Fig. 2). 
The main discussion, in that paper, centred around the ability of each transmitter type to discriminate 2D (with a given strike) and 3D ore bodies.

To reiterate, more accurately, the cross-sectional field behaviour of the transmitters, the modulus of the total electric field from (a) a y-directed HMD and (b) a VMD is shown contoured in Figure 3 across both half-spaces. The calculation uses a unit dipole moment $(1 \mathrm{~A} / \mathrm{m})$ with the transmitter at a height of $40 \mathrm{~m}$ above a conductive half-space of $10 \Omega \mathrm{m}$. The frequency is $3 \mathrm{kHz}$ and the transmitter is a point dipole above the origin. The cross-sections are shown in true scale using the same (logarithmic) contour interval. Infill has been used for values $>1(\mu \mathrm{V} / \mathrm{m})$ to emphasise the subsurface areas of maximum current. The results, which display the refractive behaviour of the electric field at the surface of the half-space, can be compared with the schematic forms in Fraser (1979) and Kovacs et al. (1995).

Surface footprints are best presented in plan view. Liu and Becker (1990, Figure 1) presented footprints for the case of a perfect conductor. Here, the calculation has been undertaken using the same source and half-space parameters as above. The contoured values of the modulus of the at-surface horizontal electric field are shown in Figure 4 for a y-polarised HMD source (Figure 4a) and a VMD source (Figure 4b). The results are shown in true scale using the same (logarithmic) contour interval. The letter $M$ is used to denote the maximum electric field. For the HMD, the well-known asymmetric current distribution is revealed, elongate perpendicular to the direction of polarisation. The maximum electric field lies directly under the transmitter. If both the moment of the HMD and the flight path are parallel (the most common arrangement), then the footprint has a long axis perpendicular to the flight direction with two symmetric minima parallel to the flight direction. In contrast, the VMD provides a radially symmetric current distribution with a very localised minimum directly beneath the transmitter. The maximum electric field is a circle as shown in Figure $4 \mathrm{~b}$. The radius of the circle is $26 \mathrm{~m}$ for the transmitter height of $40 \mathrm{~m}$. In the sea-water (perfect conductor) analysis of Liu and Becker (1990), the radius of the maximum was always half the sensor height. 
Skin distances from the maximum value are shown as gray-scale zones in Figure 4. For the HMD, the skin distance defines a bilaterally symmetric shape around the central maximum. The two skin distances occur at $53 \mathrm{~m}$ (in $\mathrm{x}$ ) and $24 \mathrm{~m}$ (in y). For the VMD, two skin distances are involved; outward and inward from the circle of maximum field. The outer skin distance occurs at a radius of $74 \mathrm{~m}$. The inner zone is smaller in area than the outer zone due to the highly localised nature of the central minimum below the transmitter. The spatial behaviour of the at-surface fields is better understood when the field is rendered as a surface (Figure 5a). The skin distance is shown by a heavy line and is seen to enclose an inverted saddle shape. The saddle zone is confined within the two minima that occur parallel to the direction of polarisation. Figure $5 b$ shows the results obtained from the same model using a transmitter altitude of $90 \mathrm{~m}$. The geometrical broadening of both the induced field and the skin distance footprint is very evident. Figure 6a shows the same VMD $40 \mathrm{~m}$ data, as in Figure 4b, rendered as a surface, with the $90 \mathrm{~m}$ altitude data shown in Figure $6 \mathrm{~b}$. In these Figures the inner skin distance is concealed within the funnel of the central minimum. The outer skin distance is a circle defined by the heavy line and is seen to extend towards a radius of $150 \mathrm{~m}$ in the case of the $90 \mathrm{~m}$ altitude data.

\section{FOOTPRINTS AS A FUNCTION OF ALTITUDE}

It should be evident, from the results presented thus far, that a transmitter footprint, defined in terms of electromagnetic skin distance, is a volumetric parameter. The parameter is made up of two principal parts. The first part is an atsurface current distribution which is geometrically imposed by the transmitter orientation and height as illustrated in Figure 3. The at-surface current distribution defines the maximum lateral scale of the footprint. The second part of the volumetric parameter is a skin distance surface that defines the attenuation of the at-surface, non-planar electric field within the earth. This second component is controlled by both resistivity and frequency. The second component relates to the effective penetration depth of the measurement and approximate transforms to 
estimate such a 'centroid depth' from survey data have been developed (Sengpiel, 1988; Siemon, 2001).

In order to arrive at a useful analysis of AEM footprints, the at-surface skin distances have been analysed as a function of transmitter altitude. The effect has a primary dependence on altitude and a secondary dependence on both resistivity and frequency. The behaviour is studied across the altitude range from $20 \mathrm{~m}$ to $100 \mathrm{~m}$ thus taking in the typical range of towed-bird and fixed-wing survey flight heights.

The behaviour of the at-surface skin distances shown in Figure 4 suggests that an analysis of two principal scales for each of the transmitter orientations would be useful. In the case of the asymmetric HMD footprint we define a long skin distance (LSD) perpendicular to the axis of polarisation (e.g. the y-direction) and a short skin distance (SSD) along the axis of polarisation (e.g. the $x$-direction). The definitions are illustrated in Figure 7a. In the case of the symmetric VMD footprint, shown in Figure 7b, two skin distances (labelled A and B) are defined in relation to the position of maximum current. For simplicity it is better to define the scale of the footprint from the origin (directly beneath the transmitter). Two radii (MAX and OSD) are defined as shown in Figure $7 \mathrm{~b}$. The first radius defines the position of the maximum current (MAX) and the second defines the outer skin distance (OSD). In conventional (plane wave) terms, the annulus formed by the subtraction of the MAX radius from the OSD radius ( $A$ in Figure $7 b$ ) would define a principal skin distance zone however the form of the VMD also provides an inner skin distance. Due to the highly localised nature of the central minimum (Figures $4 \mathrm{~b}$ and 6 ), the inner skin distance ( $B$ in Figure $7 b$ ) always extends very close to the origin. This suggests that the principal effective skin distances, when considering the VMD footprint, may be defined using the radius of the maximum current together with the radius of the outer skin distance.

The study has been conducted using two half-space resistivities of $10 \Omega \mathrm{m}$ and 1000 $\Omega \mathrm{m}$. The former is taken to represent moderately conductive environments and the latter resistive environments. For each of these models, the analysis has been conducted at the three frequencies of 1,10 and $100 \mathrm{kHz}$. 


\section{HMD results}

Footprint parameters for the HMD are the short skin distance (SSD) and long skin distance (LSD). The results obtained for half-space resistivities of 10 and $1000 \Omega \mathrm{m}$ are shown in Figures $8 \mathrm{a}$ and 8b, respectively. Strong quasi-linear behaviour is evident at the lower frequencies of 1 and $10 \mathrm{kHz}$. The overall dependence of skin distance on both frequency and resistivity is as expected from conventional (plane wave) behaviour. Low frequencies and high resistivities are seen to provide the largest skin distances.

\section{VMD results}

Footprint parameters for the VMD are the radius of the maximum current (MAX) and the outer skin distance (OSD). The results obtained for half-space resistivities of 10 and $1000 \Omega m$ are shown in Figures 9a and 9b, respectively. In order to accommodate the large outer skin distances, the distance axis has been extended to $200 \mathrm{~m}$.

The maximum lateral scales of the two footprint types are defined as the LSD in the case of the HMD and the OSD in the case of the VMD. As in the case of the previous studies, it is apparent that the HMD provides a smaller footprint than the VMD. In the case of a survey altitude of $30 \mathrm{~m}$ (typical towed-bird), the HMD provides a maximum footprint of $44 \mathrm{~m}$ while the VMD provides a footprint of $64 \mathrm{~m}$ in a conductive (10 $\Omega \mathrm{m}$ ) environment at low (1 kHz) frequency. These figures change only slightly in a more resistive $(1000 \Omega m)$ environment. The HMD maximum footprint increases to $47.5 \mathrm{~m}$ and the VMD maximum footprint increases to $70 \mathrm{~m}$.

The differences in the footprints for the two orientations are far less pronounced than in the previous studies. The study of Liu and Becker (1990) indicated a square with sides of $40.5 \mathrm{~m}$ for the HMD (vertical coaxial coils) and a square with sides of 
$112 \mathrm{~m}$ for the VMD (horizontal coplanar coils), at a height of $30 \mathrm{~m}$. As well as the limitations imposed by a perfect conductor, Kovacs et al. (1995) also noted a limitation in the use of a rectilinear averaging zone, when the induced current distribution is not rectilinear. The authors suggested that their footprint/altitude results may be overestimated and this appears to be the case.

We can also consider the question as to the appropriate maximum sensor height to maintain a minimum footprint of (say) $<100 \mathrm{~m}$. This question constitutes a method of maintaining a high resolution focus during survey operations. Here we restrict ourselves to the more practical frequencies of 1 and $10 \mathrm{kHz}$. Using the results of Figure 8, for the HMD LSD, the maximum height is found to be $93 \mathrm{~m}$ at $10 \mathrm{kHz}$ and 77 $\mathrm{m}$ at $1 \mathrm{kHz}$ in a conductive (10 $\Omega \mathrm{m})$ environment, reducing to $66 \mathrm{~m}$ and $64 \mathrm{~m}$ in a resistive (1000 $\Omega \mathrm{m}$ ) environment. Using the VMD OSD results of Figure 9, the maximum height is found to be $66 \mathrm{~m}$ in a conductive $(10 \Omega \mathrm{m})$ environment, reducing to $43 \mathrm{~m}$ in a resistive $(1000 \Omega \mathrm{m})$ environment. It can be concluded that a specific degree of resolution can be maintained when fixed wing surveys, using HMD transmitters, are operated at higher altitudes than systems employing only VMD transmitters.

Finally, it is possible to establish adequate linear relationships between the skin distance parameters and elevation for the $10 \mathrm{kHz}$ data shown in Figures 8 and 9. For the HMD, linear relationships have been obtained for the MAX and OSD parameters at $10 \mathrm{kHz}$. For the VMD, linear relationships have been calculated for the SSD and LSD parameters at $10 \mathrm{kHz}$. The least-squares parameters for the fit: $y=a_{0}+a_{1} \cdot x$, where $\mathrm{y}$ denotes a skin distance parameter and $\mathrm{x}$ is transmitter elevation are given in Table 1. The coefficients obtained offer a means of predicting skin distance footprint sizes for mid-frequency $(10 \mathrm{kHz})$ AEM data sets over the altitude range from 20 to $100 \mathrm{~m}$.

In previous studies (Liu and Becker, 1990), a vertical coaxial system (using a HMD transmitter) was found to provide a footprint/altitude ratio of 1.35 and the ratio increased to 3.73 for a horizontal coplanar system (a VMD system). Strictly speaking, 
the ratios only apply at the inductive limit and for a coil-coil separation of $6.5 \mathrm{~m}$. In the present study the corresponding maximum (long skin distance) HMD ratios are found to be between 0.987 for a $10 \Omega \mathrm{m}$ half-space and between 1.425 for a 1000 $\Omega m$ half-space. The VMD ratios (using the outer skin distance) are found to be between 1.313 for a $10 \Omega \mathrm{m}$ half-space and 2.096 for a $1000 \Omega \mathrm{m}$ half-space. Clearly the estimates of the footprint scales for a VMD system can be revised downward from previous estimates.

\section{DISCUSSION}

As AEM studies move to more detailed assessments of the near-surface, a more precise knowledge of the behaviour of the footprints, and hence the spatial averages involved in the measurements becomes important. When, as here, an analysis is conducted using a finitely conducting half space, the footprint is a volume. In order to illustrate the issues, typical transmitter footprint volumes obtained from a low elevation (30 m) analysis are shown in Figure 10a (a HMD) and 10b (a VMD). The frequency used is $10 \mathrm{kHz}$ and the half-space resistivity is $100 \Omega \mathrm{m}$. The 3D contoured volume is defined, as previously, as 1 skin distance from the maximum amplitude. The SE quadrant of the image has been made transparent to aid visualisation. Maximum electric field amplitudes occur at the origin in the case of the HMD (Figure 10a) and along a radius of $21 \mathrm{~m}$ in the case of the VMD (Figure 10b). The horizontal scale is $200 \times 200 \mathrm{~m}$ while the vertical scale is only $20 \mathrm{~m}$. To obtain a realistic impression of the true scale footprint, one would have to imagine the same images compressed by a factor of 4 along the vertical axis.

Since only a limited number of AEM systems exist, the bandwidth and frequencies that can be used on a given survey are, more or less, fixed. The available frequencies effectively determine the depth of penetration, whatever the chosen environment. In terms of survey planning, however, choices related to flight altitude and flight line spacing can be critical in terms of both target discrimination and cost. The choice of flight altitude in populated areas, is likely to be controlled by regulatory authorities 
and thus may be predefined. In some cases the regulatory restrictions will preclude low level surveys; this in turn may rule out the use of towed bird systems on signal/noise grounds. The choice of optimum flight line spacing, for a given survey altitude, still remains a key issue.

The skin distance volumes shown in Figure 10 relate to low level $(30 \mathrm{~m})$ surveying. Depending on air speed and data sampling rate, the distance sampling interval along each flight line may range from 3 to $4 \mathrm{~m}$ for HEM and from 10 to $15 \mathrm{~m}$ for a fixedwing survey. The planar upper surface of the volume shows north-south flight lines spaced at $50 \mathrm{~m}$ intervals (dotted lines with arrows). For the model parameters used

it could be argued that the more compact footprint of the HMD indicates a requirement for a tighter line spacing (say $50 \mathrm{~m}$ ) than that of the VMD where a flight line spacing of $100 \mathrm{~m}$ may be adequate. In the case of a bird containing transmitters of both orientations, the survey design must balance both resolution and cost.

\section{CONCLUSIONS}

Existing estimates of AEM footprints have a restricted application range since they use a perfectly conducting thin sheet to model the induced current distribution. In addition, published results are system specific so that they depend on the coil separation which differs substantially between towed bird and fixed wing systems.

An alternative study has been conducted using a finitely conducting half-space. The footprint, in terms of this form of modelling (3D source and 1D earth), actually becomes a volume in the lower half-space. The maximum electric fields are produced at the surface and are geometrically imposed by the orientation and altitude of the transmitter. Within the earth these at-surface fields attenuate and form a volumetric footprint (with maximum lateral extent at the surface) that fully defines the principal spatial sensitivity zone of the measurement. It is this principal volume that will determine the extent to which any $2 \mathrm{D}$ and $3 \mathrm{D}$ variations in resistivity structure, at a given physical scale, will interact with the measurement. 
Although the footprint obtained is a volumetric quantity, only the at-surface, lateral footprints have been analysed in detail. In order to remove the system dependence of previous studies, a transmitter footprint has been defined in terms of electromagnetic skin distance; this has the same attributes as the conventional skin depth of an electromagnetic field. The problem is also simplified in that only HMD and VMD transmitter systems need to be considered. The degree of coupling, and other signal/noise issues, can be considered separately to that of the footprint scale.

Electromagnetic skin distances, two for each of the coil orientations, have been defined. These at-surface footprints have been calculated as a function of transmitter altitude. The behaviour has a primary dependence on altitude and a secondary dependence on resistivity and frequency. The behaviour has been studied across an altitude range from 20 to $100 \mathrm{~m}$ and therefore the results allow for both towed bird and fixed wing survey assessments. The analysis covers an extensive frequency range and results have been obtained for two specific resistivity values that represent a range from conductive $(10 \Omega \mathrm{m})$ to resistive $(1000 \Omega \mathrm{m})$ environments.

The footprints, as defined here, display a quasi-linear behaviour with altitude, particularly for the mid-range frequency of $10 \mathrm{kHz}$. This behaviour has enabled the coefficients of a linear, least-squares relationship to be obtained thus assisting with the prediction of footprint scales. The footprint estimates obtained in the present study imply that a HMD system retains a smaller footprint, by a factor of between 1.3 and 1.5 than a VMD system operated at the same height. The results imply that a specific degree of resolution can be maintained when fixed wing surveys, using HMD transmitters, are operated at higher altitudes than systems employing only VMD transmitters. This may be advantageous in cases where regulations preclude surveying at low flight altitudes. 


\section{ACKNOWLEDGEMENTS}

The author thanks reviewers James Reid, Jonathan Nyquist and Richard Smith together with the Associate Editor for useful comments and advice. This report is published with the permission of the Executive Director, British Geological Survey (NERC).

\section{REFERENCES}

Anderson, W.L. 1979. Numerical integration of related Hankel transforms of order 0 and 1 by adaptive digital filtering. Geophysics 44, 1287-1305.

Beamish, D. and Kurimo, M. 2000. Trial airborne EM surveys to assess minewater pollution in the UK. 62 ${ }^{\text {nd }}$ EAGE Conference and Technical Exhibition, Glasgow, UK. Extended Abstracts, Vol. 1, D-22.

Beard, L.P. and Lutro, O. 2000. Airborne geophysics and infrastructure planning - a case study, Journal of Environmental and Engineering Geophysics 5, 1-10.

Christensen, N.B. 1990. Optimized fast Hankel transform filters. Geophysical Prospecting 38, 545-568.

Doll, W.E., Nyquist, J.E., Beard, L.P. and Gamey, T.J. 2000. Airborne geophysical surveying for hazardous waste site characterisation on the Oak Ridge Reservation, Tennessee. Geophysics 65, 1372-1387.

Fitterman, D.V. and Deszcz-Pan, M. 1998. Helicopter EM mapping of saltwater intrusion in Everglades National Park. Exploration Geophysics 29, 240-243.

Fraser, D.C. 1979. The multicoil II airborne electromagnetic system. Geophysics 44, 1367-1394.

Frischknecht, F.C. 1967. Fields about an oscillating magnetic dipole over a two-layer earth and application to ground and airborne electromagnetic surveys. Colorado School of Mines Quarterly 62, 326pp.

Jokinen, T. and Lanne, E. 1996. Airborne geophysics in mapping contaminant plumes from landfills. SAGEEP Extended Abstracts, Keystone, Colorado, 981-995. 
Kirsch, R., Sengpiel, K.-P., and Voss, W. 2001. Aquifer protection by vulnerability mapping - a geophysical approach. European Journal of Environmental and Engineering Geophysics, submitted.

Kovacs, A., Holladay, J.S. and Bergeron Jr., C.J. 1995. The footprint/altitude ratio for helicopter electromagnetic sounding of sea-ice thickness: Comparison of theoretical and field estimates. Geophysics 60, 374-380.

Liu, G. and Becker, A. 1990. Two-dimensional mapping of sea-ice keels with airborne electromagnetics. Geophysics 55, 239-248.

Poikonen, A., Sulkanen, K, Oksama, M., and Suppala, I. 1998. Novel dual frequency fixed wing airborne EM system of Geological Survey of Finland (GTK). Exploration Geophysics 29, 46-51.

Puranen, R., Säävuori, H., Sahala, L., Suppala, I., Mäkilä, M. and Lerssi, J. 1999. Airborne electromagnetic mapping of surficial deposits in Finland. First Break, May 1999, 145-154.

Reid J.E., and Macnae, J.C. 1999. Doubling the effective skin depth with a local source. Geophysics 64, 732-738.

Sengpiel, K.-P. 1988. Approximate inversion of airborne EM data from a multilayered ground. Geophysical Prospecting 36, 446-459.

Siemon, B. 2001. Improved and new resistivity-depth profiles for helicopter electromagnetic data. Journal of Applied Geophysics 46, 65-76.

Spies, B.R. 1989. Depth of investigation in electromagnetic sounding methods. Geophysics 54, 872-888.

Wait, J.R. 1954. Mutual coupling of loops lying on the ground. Geophysics 19, 290296.

Wait, J.R. 1955. Mutual electromagnetic coupling of loops over a homogenous ground. Geophysics 20, 630-637.

Ward, S.H. 1967. Electromagnetic theory for geophysical applications. In: Mining geophysics, Vol. II, Theory (eds. D.A. Hansen, W.E. Heinrichs, R.C. Holmer, R.E. MacDougall, G.R. Rogers, J.S. Dumner and S.H. Ward), pp. 10-196. Soc. Expl. Geophys, Tulsa, OK. 
Table 1 The least-squares parameters of the fit: $y=a_{0}+a_{1} \cdot x$, where $y$ denotes a skin distance parameter $(\mathrm{m})$ and $\mathrm{x}$ is transmitter elevation $(\mathrm{m})$. Skin distance parameters are the short skin distance (SSD) and long skin distance (LSD) for the vertical magnetic dipole (VMD). Skin distance parameters are the radius of the maximum current (MAX) and the outer skin distance (OSD) for a horizontal magnetic dipole (HMD). The parameters were obtained for a frequency of $10 \mathrm{kHz}$ and for two half space resistivities (10 and $1000 \Omega \mathrm{m}$ ).

\begin{tabular}{|l|l|l|l|l|}
\hline Resistivity $\Omega \mathrm{m}$ & Dipole Orientation & Skin parameter & $\mathrm{a}_{0}$ & $\mathrm{a}_{1}$ \\
\hline 10 & HMD & SSD & 3.414 & 0.466 \\
\hline 10 & HMD & LSD & 7.694 & 0.987 \\
\hline 10 & VMD & MAX & 3.255 & 0.522 \\
\hline 10 & VMD & OSD & 12.533 & 1.313 \\
\hline 1000 & HMD & SSD & 1.561 & 0.642 \\
\hline 1000 & HMD & LSD & 5.000 & 1.425 \\
\hline 1000 & VMD & MAX & 0.289 & 0.693 \\
\hline 1000 & VMD & OSD & 7.694 & 2.096 \\
\hline
\end{tabular}




\section{FIGURE CAPTIONS}

Figure 1 Schematic of magnetic dipole coil-coil systems described in the text. Rings denote coils and arrows denote dipole polarisation. (a) HMD system, vertical coaxial coils. (b) HMD system, vertical coplanar coils. (c) VMD system, horizontal coplanar coils.

Figure 2 Real (Re) and Imaginary $(\mathrm{Im})$ coupling ratios of the three coil systems (a to c) shown in Figure 1 as a function of half-space resistivity. Calculated at a frequency of $3 \mathrm{kHz}$ and an elevation of $40 \mathrm{~m}$. Two sets of curves are shown for coil separations of $6 \mathrm{~m}$ (lower set) and $20 \mathrm{~m}$ (upper set).

Figure 3 Modulus of the horizontal electric field $(\mu \mathrm{V} / \mathrm{m})$ contoured in the $x-z$ plane due to: (a) a HMD polarised in the y-direction (into the page), (b) a VMD polarised in the z-direction. Each source has a dipole moment of $1 \mathrm{~A} / \mathrm{m}$, a frequencyof $3 \mathrm{kHz}$ and is $40 \mathrm{~m}$ above a $10 \Omega \mathrm{m}$ half-space. The results are plotted at a true scale using a logarithmic interval. Contours with infill denote values $>1(\mu \mathrm{V} / \mathrm{m})$.

Figure $4 \quad$ Modulus of the horizontal electric field $(\mu \mathrm{V} / \mathrm{m})$ contoured in the $x-y$ plane due to: (a) a HMD polarised in the y-direction, (b) a VMD polarised in the $z$ direction. Each source has a dipole moment of $1 \mathrm{~A} / \mathrm{m}$, a frequency of $3 \mathrm{kHz}$ and is 40 $\mathrm{m}$ above a $10 \Omega \mathrm{m}$ half-space. The results are plotted at a true scale using a logarithmic interval. Infill denotes the area where the electric field is greater than $1 / \mathrm{e}$ of the maximum. The letter $\mathrm{M}$ denotes maximum current.

Figure 5 Modulus of the horizontal electric field contoured as a surface in the $x-y$ plane due to a HMD polarised in the $y$-direction, above the origin. The source has a dipole moment of $1 \mathrm{~A} / \mathrm{m}$, a frequency of $3 \mathrm{kHz}$ and is above a $10 \Omega \mathrm{m}$ half-space. The skin distance, defined in the text, is shown by a heavy line. (a) Dipole elevation of $40 \mathrm{~m}$. (b) Dipole elevation of $90 \mathrm{~m}$.

Figure 6 Modulus of the horizontal electric field contoured as a surface in the $x-y$ plane due to a VMD above the origin. The source has a dipole moment of $1 \mathrm{~A} / \mathrm{m}$, a frequency of $3 \mathrm{kHz}$ and is above a $10 \Omega \mathrm{m}$ half-space. The skin distance, defined in the text, is shown by a heavy line. (a) Dipole elevation of $40 \mathrm{~m}$. (b) Dipole elevation of $90 \mathrm{~m}$.

Figure 7 Illustration of skin distances. (a) A HMD, polarised in the y-direction, provides a long skin distance (LSD) perpendicular tothe axis of polarisation, typically the flight direction, and a short skin distance (SSD) along the axis of polarisation. (b) A VMD provides a maximum electric field at a radius of MAX. Two skin distances are labelled $A$ and $B$. The radial distance of the outer skin distance (OSD) is used as a parameter.

Figure $8 \quad$ Variation of short skin distance (SSD) and long skin distance (LSD) as a function of altitude for a HMD. The variation at the 3 frequencies of 1,10 and 100 
$\mathrm{kHz}$ is shown by lines with different symbols. (a) Results for a half-space resistivity of $10 \Omega \mathrm{m}$. (b) Results for a half-space resistivity of $1000 \Omega \mathrm{m}$.

Figure 9 Variation of radius of maximum electric field (MAX) and outer skin distance (OSD) as a function of altitude for a VMD. The variation at the 3 frequencies of 1,10 and $100 \mathrm{kHz}$ is shown by lines with different symbols. (a) Results for a halfspace resistivity of $10 \Omega \mathrm{m}$. (b) Results for a half-space resistivity of $1000 \Omega \mathrm{m}$.

Figure 10 Three dimensional perspective views of skin distance volumes generated by magnetic dipoles at a frequency of $10 \mathrm{kHz}, 30 \mathrm{~m}$ above a $100 \Omega \mathrm{m}$ halfspace. The volume shown $(100 \times 100 \times 20 \mathrm{~m})$ has a vertical exaggeration of $\times 4$. The SE quadrant has been cut. Dotted lines denote a flight line spacing of $50 \mathrm{~m}$. (a) HMD polarised in the y-direction, along the flight direction. (b) VMD. 

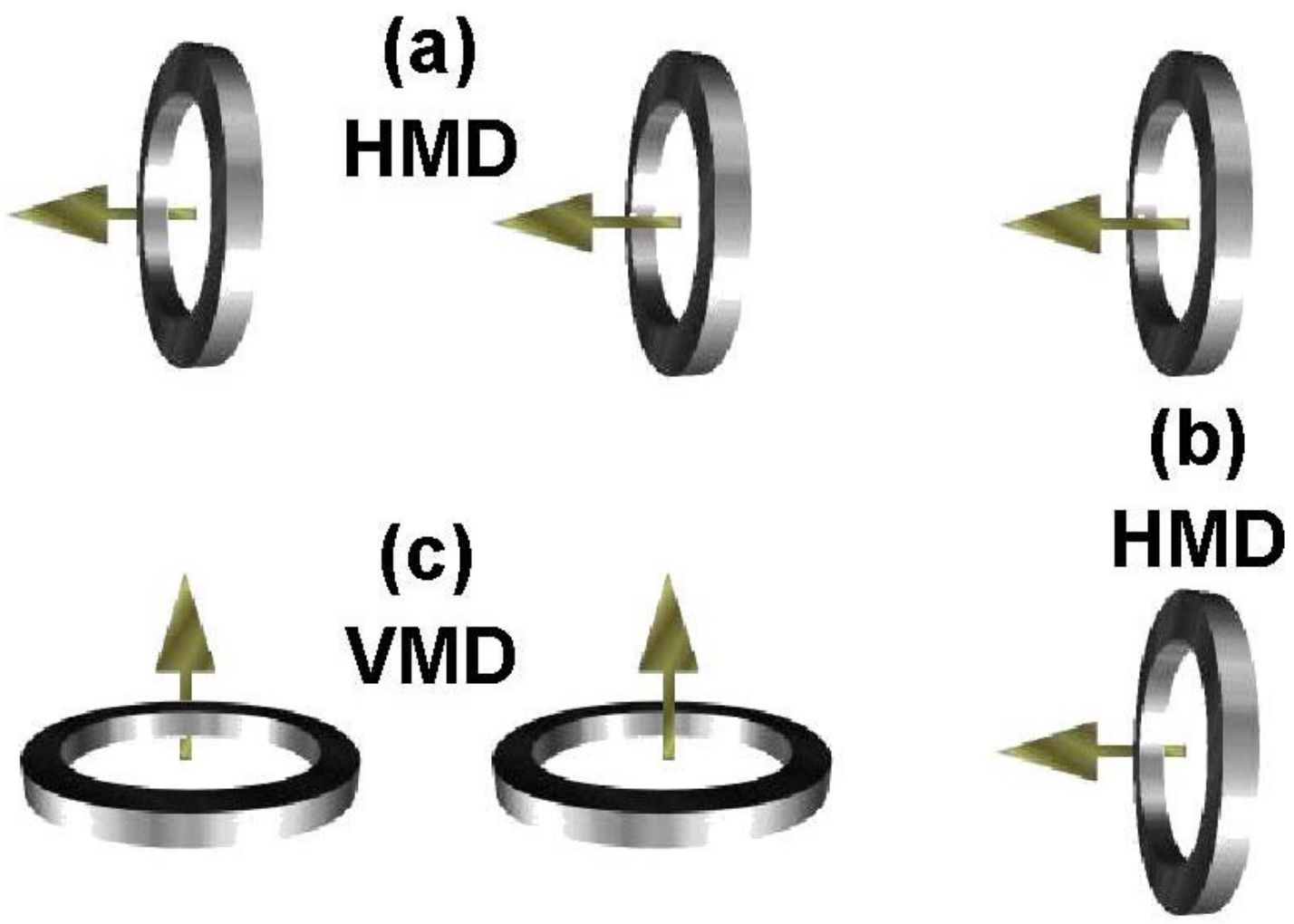

Figure 1 Schematic of magnetic dipole coil-coil systems described in the text. Rings denote coils and arrows denote dipole polarisation. (a) HMD system, vertical coaxial coils. (b) HMD system, vertical coplanar coils. (c) VMD system, horizontal coplanar coils. 


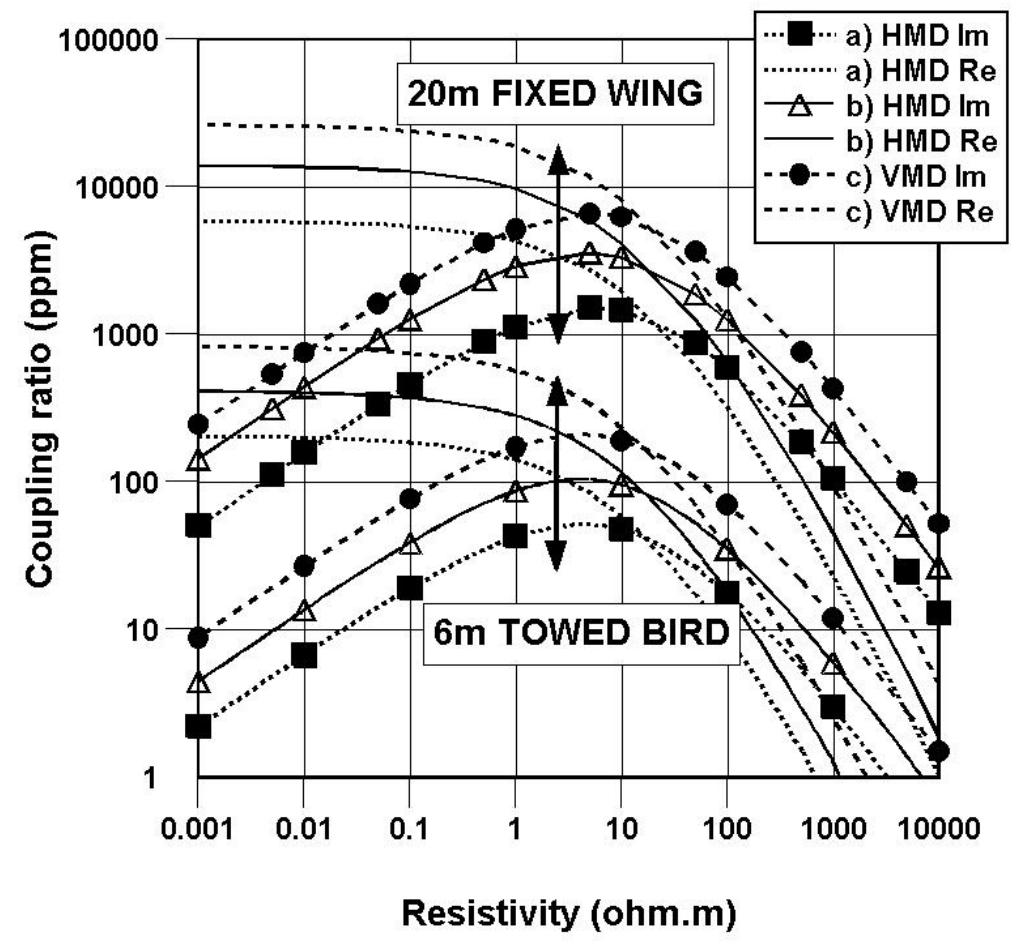

Figure 2 Real (Re) and Imaginary $(\mathrm{Im})$ coupling ratios of the three coil systems (a to c) shown in Figure 1 as a function of half-space resistivity. Calculated at a frequency of $3 \mathrm{kHz}$ and an elevation of $40 \mathrm{~m}$. Two sets of curves are shown for coil separations of $6 \mathrm{~m}$ (lower set) and $20 \mathrm{~m}$ (upper set). 

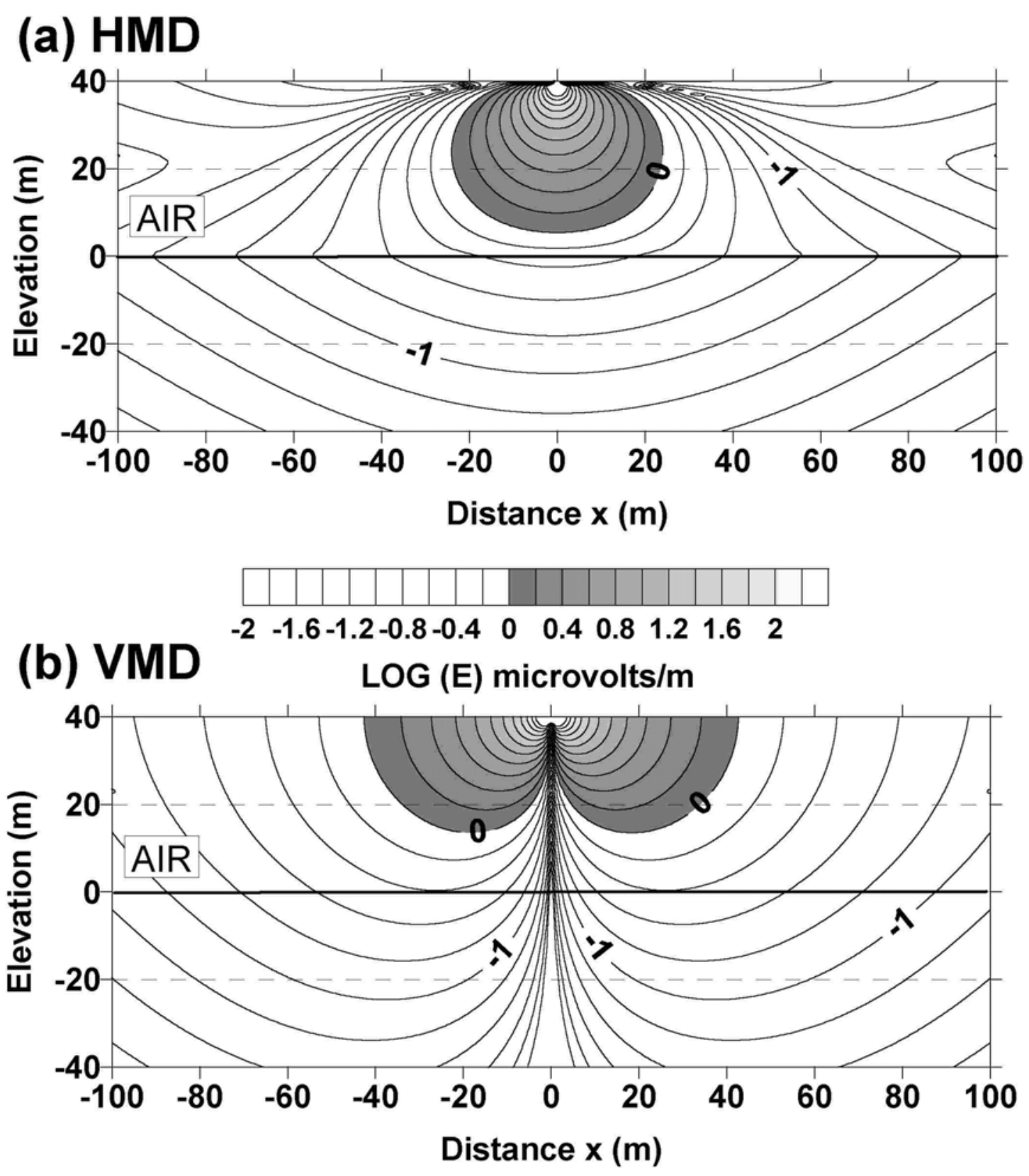

Figure 3 Modulus of the horizontal electric field $(\mu \mathrm{V} / \mathrm{m})$ contoured in the $x-z$ plane due to: (a) a HMD polarised in the $y$-direction (into the page), (b) a VMD polarised in the z-direction. Each source has a dipole moment of $1 \mathrm{~A} / \mathrm{m}, \mathrm{a}$ frequencyof $3 \mathrm{kHz}$ and is $40 \mathrm{~m}$ above a $10 \Omega \mathrm{m}$ half-space. The results are plotted at a true scale using a logarithmic interval. Contours with infill denote values $>1(\mu \mathrm{V} / \mathrm{m})$. 

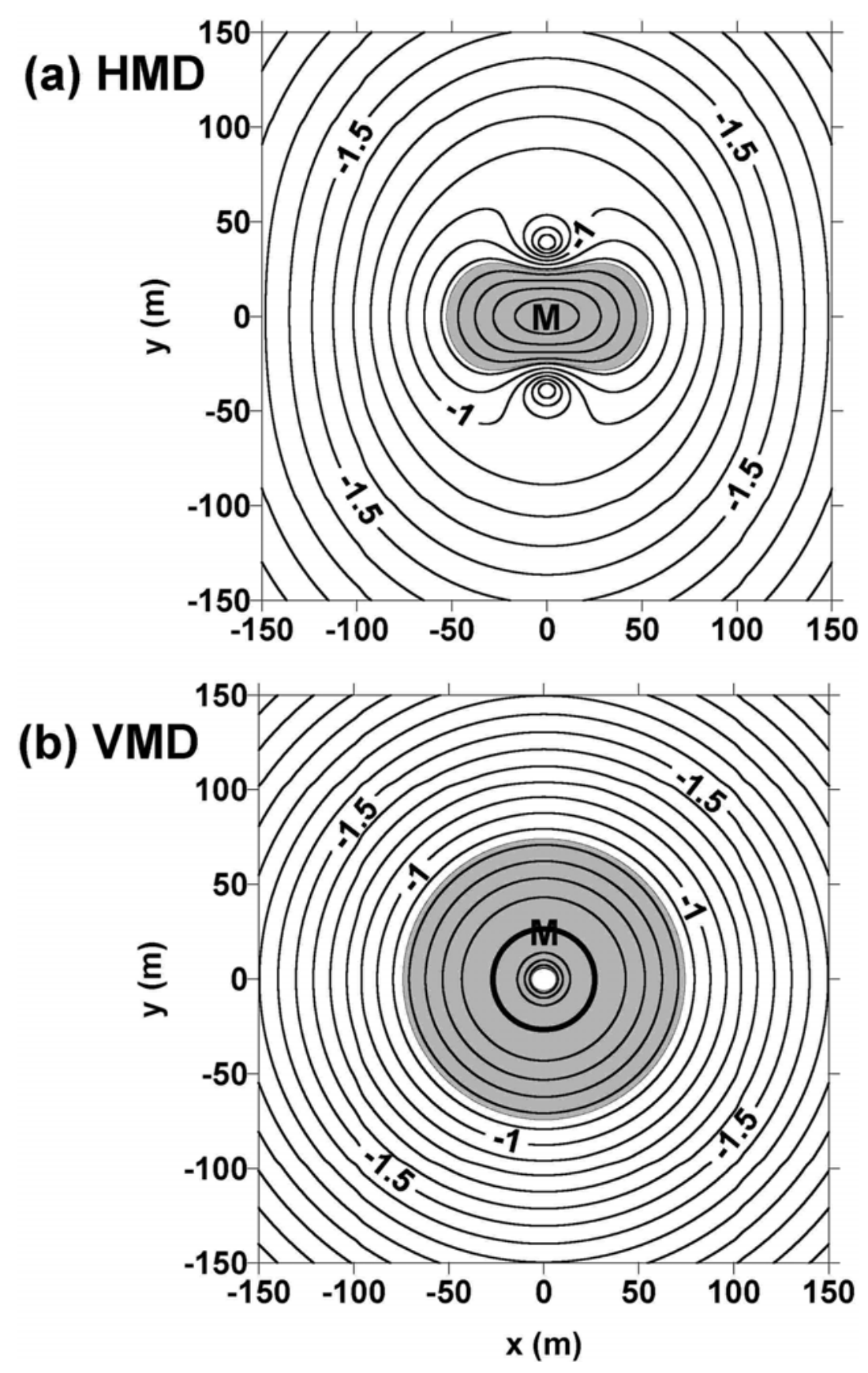

Figure $4 \quad$ Modulus of the horizontal electric field $(\mu \mathrm{V} / \mathrm{m})$ contoured in the $x-y$ plane due to: (a) a HMD polarised in the y-direction, (b) a VMD polarised in the zdirection. Each source has a dipole moment of $1 \mathrm{~A} / \mathrm{m}$, a frequency of $3 \mathrm{kHz}$ and is 40 $\mathrm{m}$ above a $10 \Omega \mathrm{m}$ half-space. The results are plotted at a true scale using a logarithmic interval. Infill denotes the area where the electric field is greater than $1 / \mathrm{e}$ of the maximum. The letter $\mathrm{M}$ denotes maximum current. 


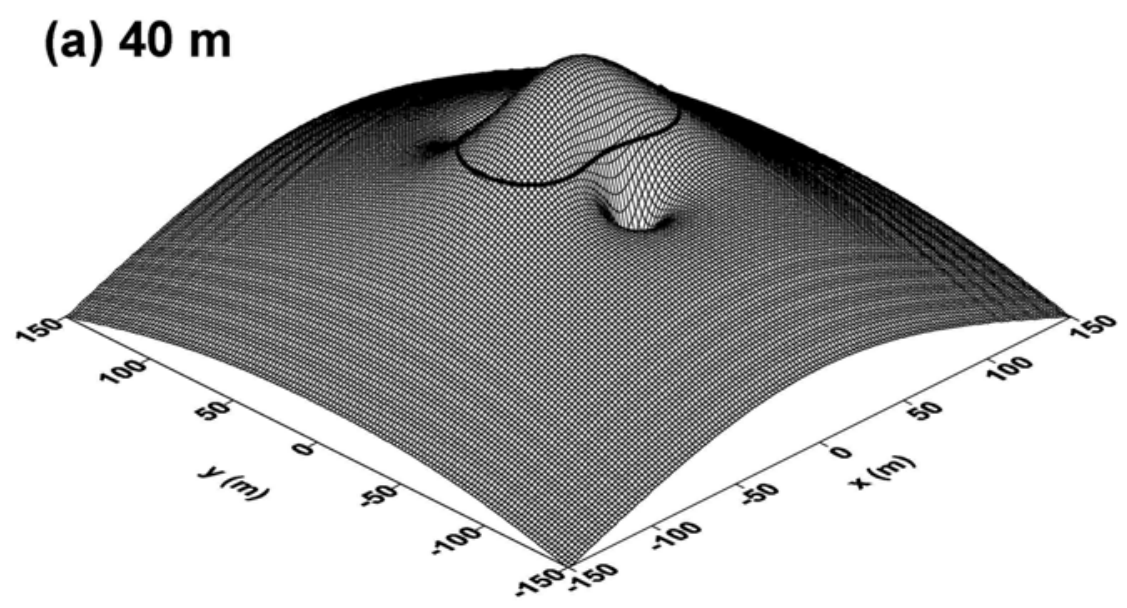

(b) $90 \mathrm{~m}$

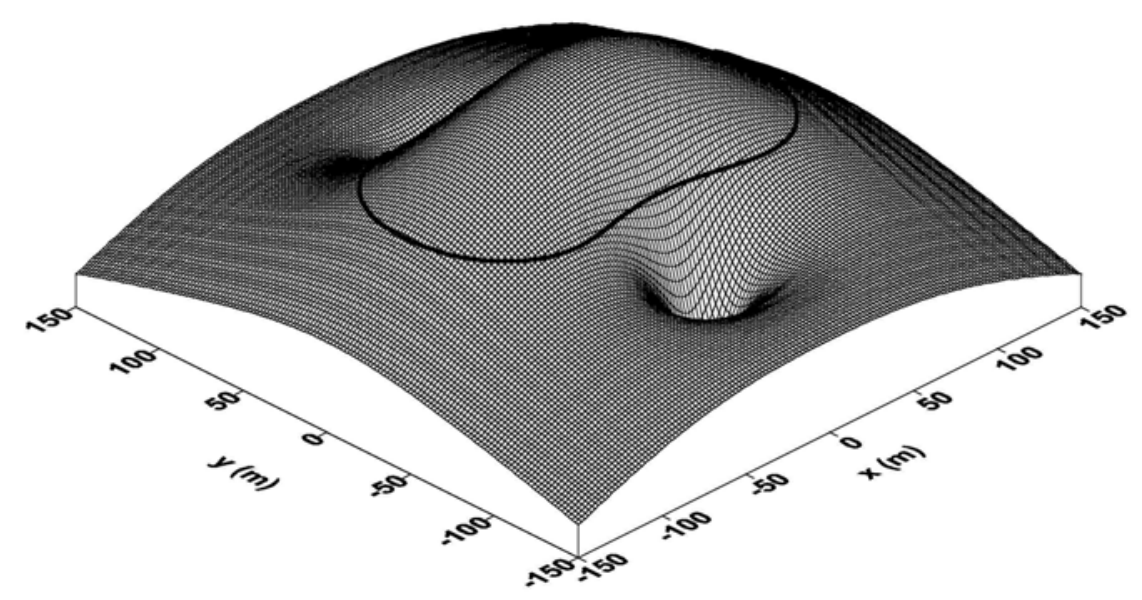

Figure $5 \quad$ Modulus of the horizontal electric field contoured as a surface in the $x-y$ plane due to a HMD polarised in the $y$-direction, above the origin. The source has a dipole moment of $1 \mathrm{~A} / \mathrm{m}$, a frequency of $3 \mathrm{kHz}$ and is above a $10 \Omega \mathrm{m}$ half-space. The skin distance, defined in the text, is shown by a heavy line. (a) Dipole elevation of $40 \mathrm{~m}$. (b) Dipole elevation of $90 \mathrm{~m}$. 

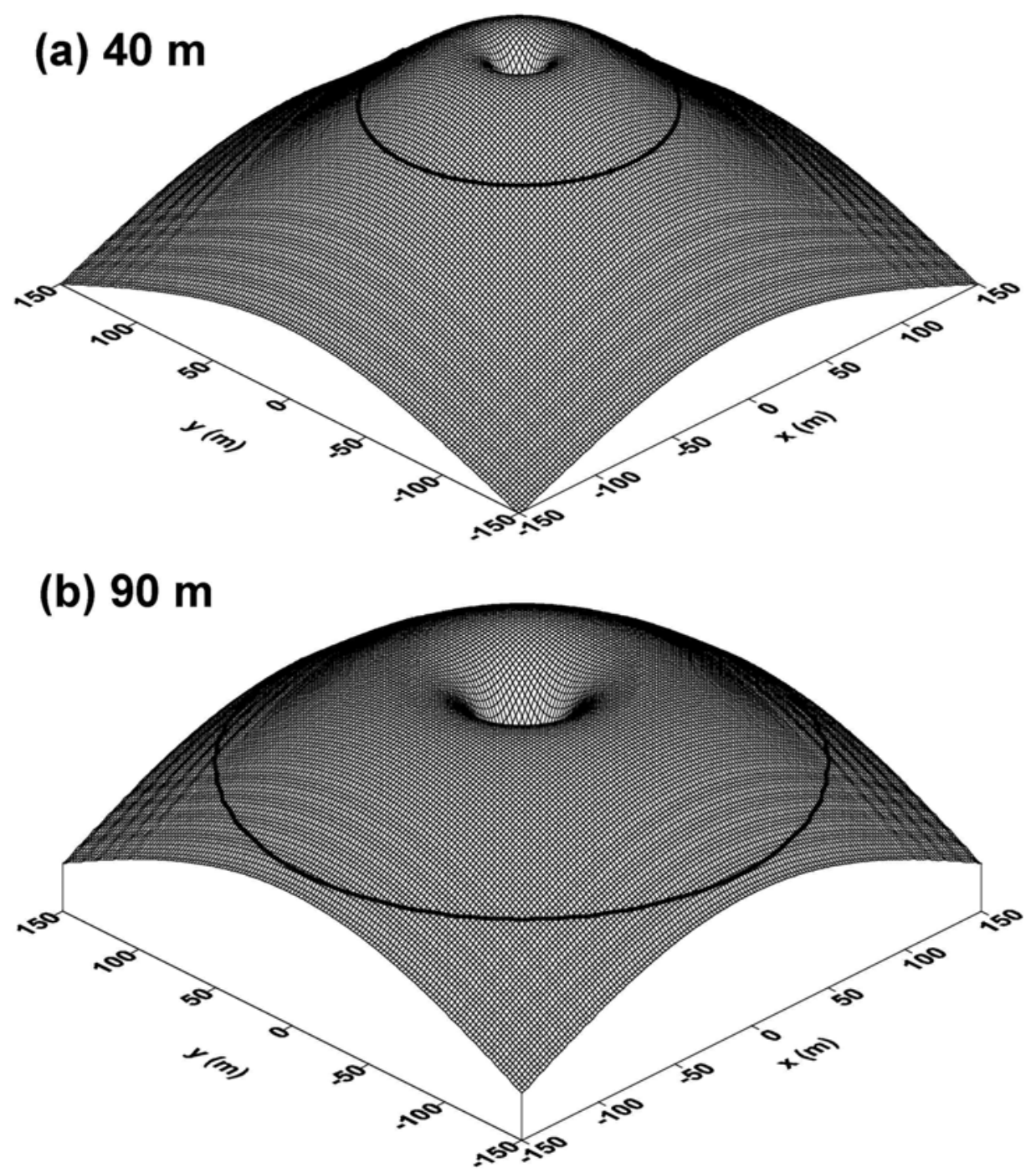

Figure 6 Modulus of the horizontal electric field contoured as a surface in the $x$-y plane due to a VMD above the origin. The source has a dipole moment of $1 \mathrm{~A} / \mathrm{m}$, a frequency of $3 \mathrm{kHz}$ and is above a $10 \Omega \mathrm{m}$ half-space. The skin distance, defined in the text, is shown by a heavy line. (a) Dipole elevation of $40 \mathrm{~m}$. (b) Dipole elevation of $90 \mathrm{~m}$. 

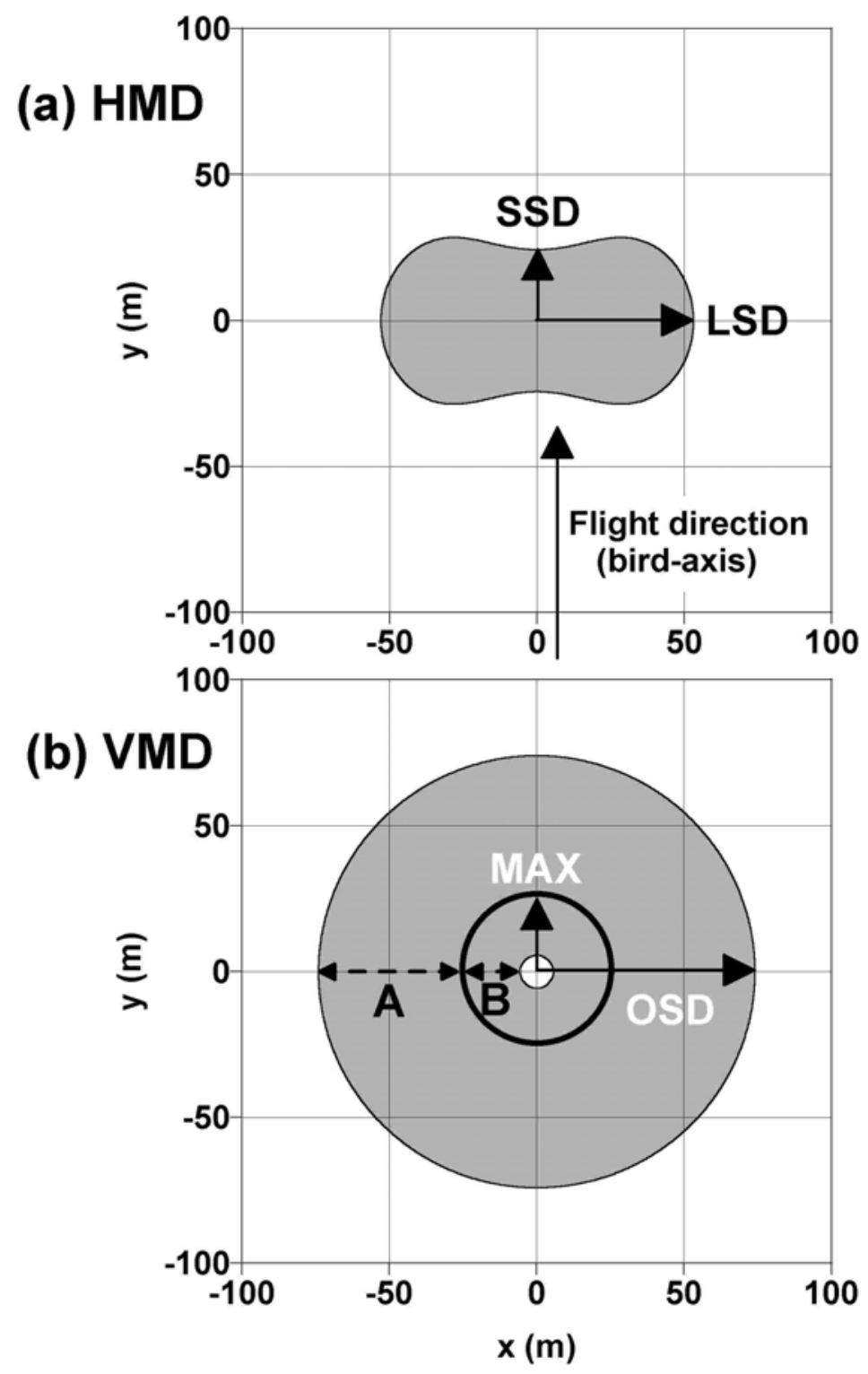

Figure 7 Illustration of skin distances. (a) A HMD, polarised in the $y$-direction, provides a long skin distance (LSD) perpendicular tothe axis of polarisation, typically the flight direction, and a short skin distance (SSD) along the axis of polarisation. (b) A VMD provides a maximum electric field at a radius of MAX. Two skin distances are labelled $A$ and $B$. The radial distance of the outer skin distance (OSD) is used as a parameter 

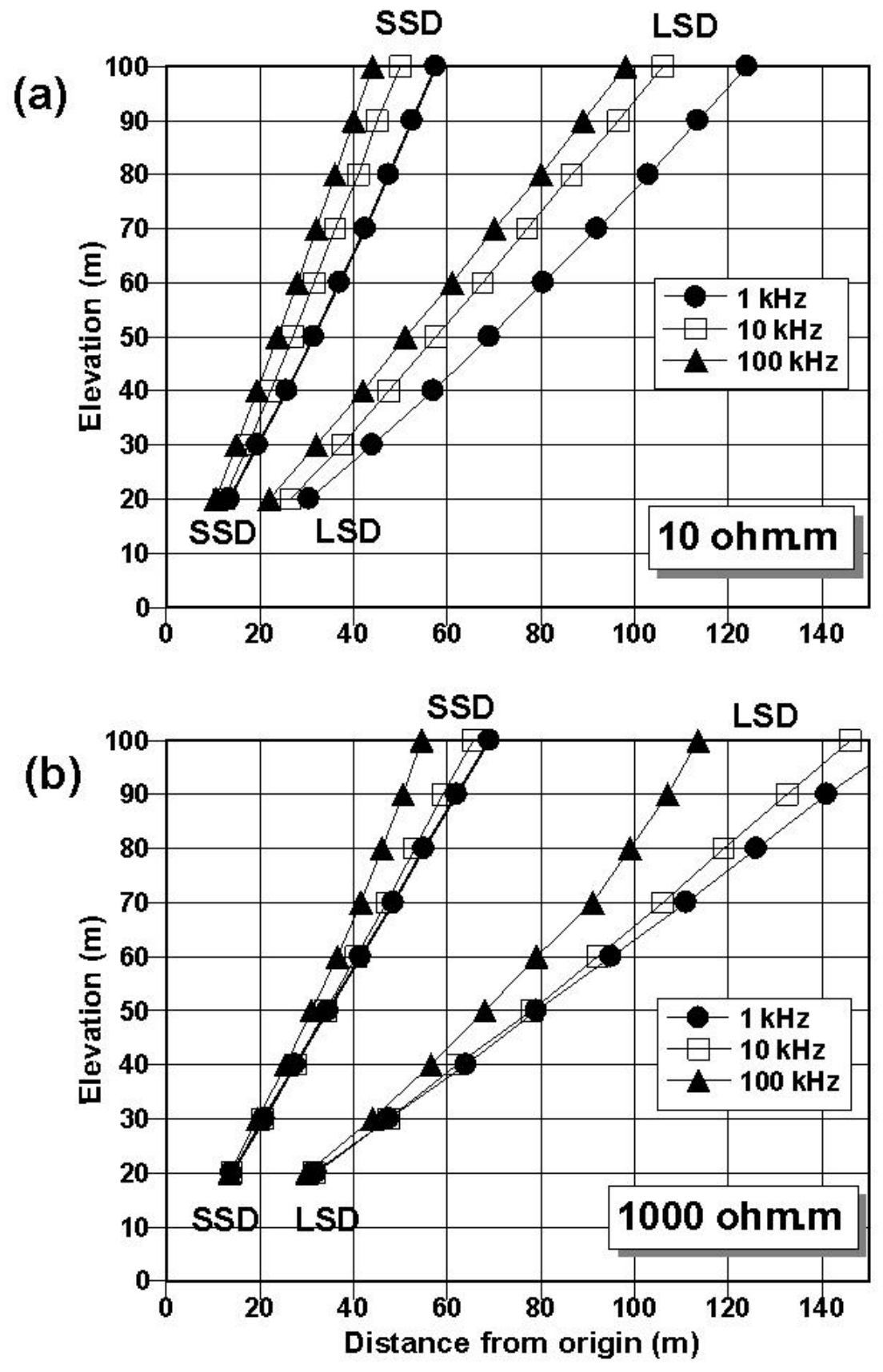

Figure $8 \quad$ Variation of short skin distance (SSD) and long skin distance (LSD) as a function of altitude for a HMD. The variation at the 3 frequencies of 1,10 and 100 $\mathrm{kHz}$ is shown by lines with different symbols. (a) Results for a half-space resistivity of $10 \Omega \mathrm{m}$. (b) Results for a half-space resistivity of $1000 \Omega \mathrm{m}$. 

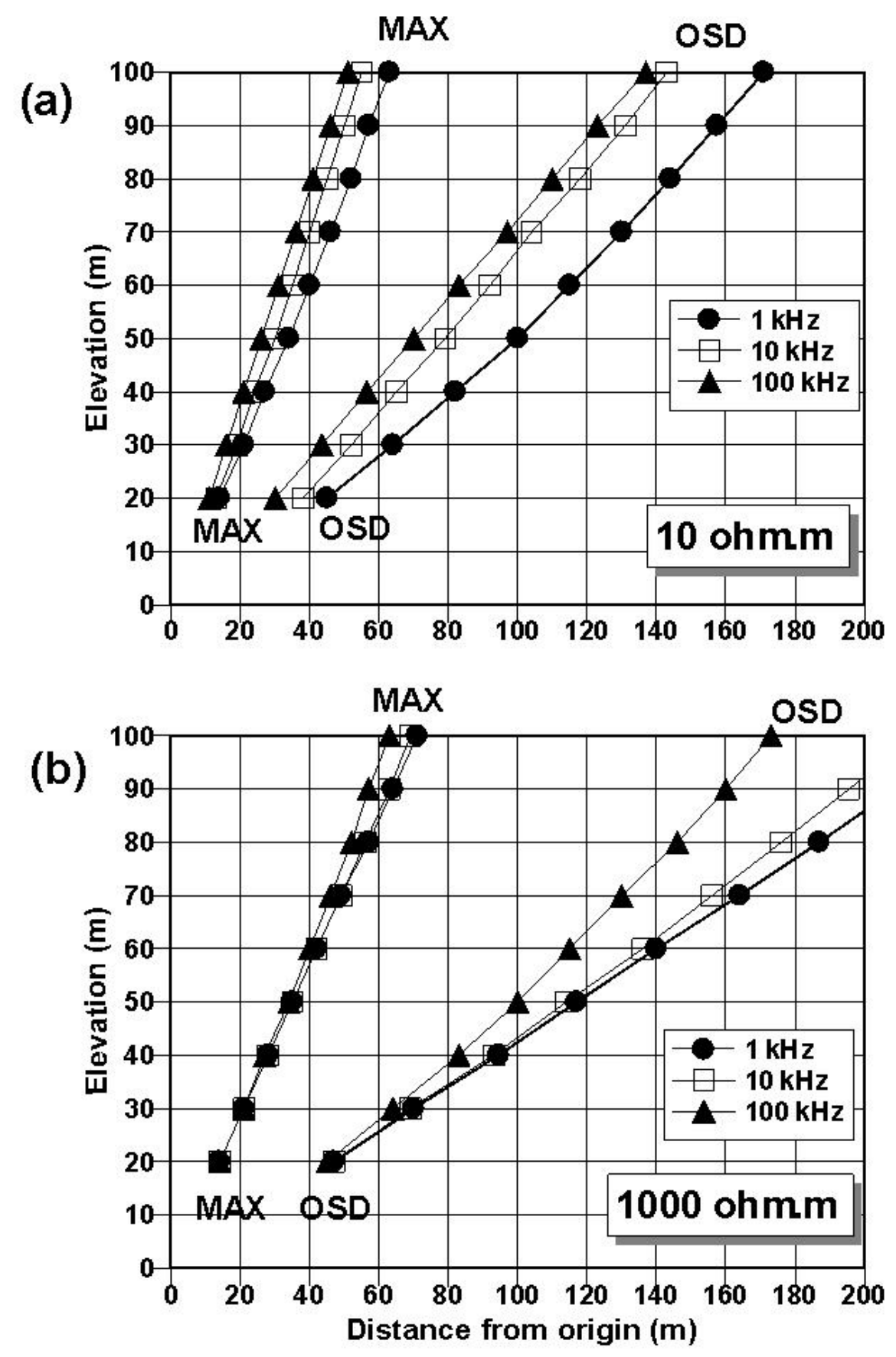

Figure 9 Variation of radius of maximum electric field (MAX) and outer skin distance (OSD) as a function of altitude for a VMD. The variation at the 3 frequencies of 1,10 and $100 \mathrm{kHz}$ is shown by lines with different symbols. (a) Results for a halfspace resistivity of $10 \Omega \mathrm{m}$. (b) Results for a half-space resistivity of $1000 \Omega \mathrm{m}$. 

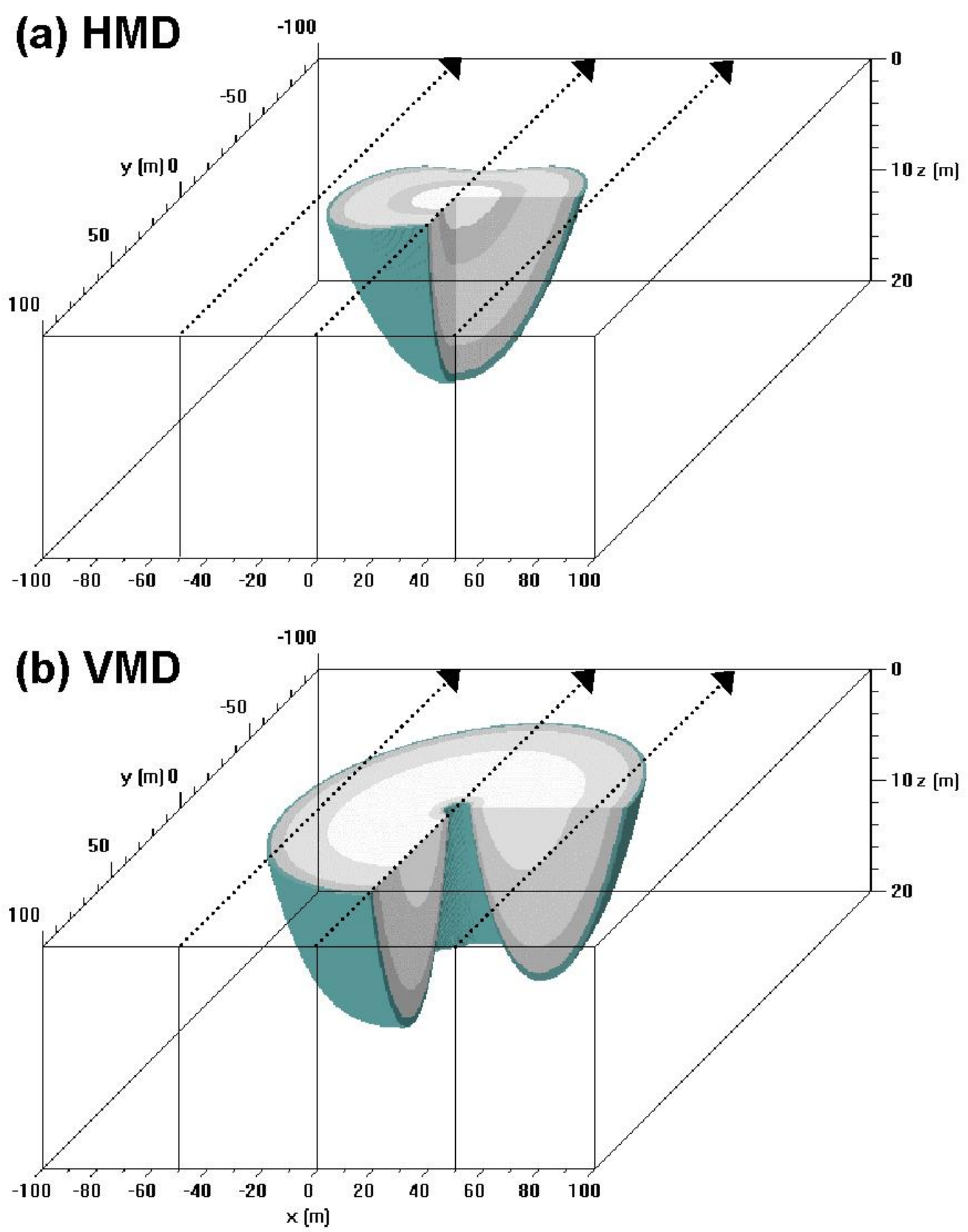

Figure 10 Three dimensional perspective views of skin distance volumes generated by magnetic dipoles at a frequency of $10 \mathrm{kHz}, 30 \mathrm{~m}$ above a $100 \Omega \mathrm{m}$ halfspace. The volume shown $(100 \times 100 \times 20 \mathrm{~m})$ has a vertical exaggeration of $\times 4$. The SE quadrant has been cut. Dotted lines denote a flight line spacing of $50 \mathrm{~m}$. (a) HMD polarised in the y-direction, along the flight direction. (b) VMD. 